Check for updates

Cite this: J. Mater. Chem. A, 2021, 9, 19741

Received 16th April 2021

Accepted 29th June 2021

DOI: $10.1039 / \mathrm{d} 1 \mathrm{ta0} 215 \mathrm{a}$

rsc.li/materials-a

\section{Chitin-amyloid synergism and their use as sustainable structural adhesives $\dagger$}

\author{
Luiz G. Greca, (D) a Kevin J. De France, (D) b Johanna Majoinen, ${ }^{a}$ Nico Kummer, (D) bc \\ Otso I. V. Luotonen, a Silvia Campioni, ${ }^{b}$ Orlando J. Rojas, (D) *ad Gustav Nyström (D)*bc \\ and Blaise L. Tardy (iD)*a
}

Structural adhesives are relevant to many engineering applications, especially those requiring load-bearing joints with high lap shear strength. Typical adhesives are synthesized from acrylics, epoxies, or urethanes, which may pose a burden to sustainability and the environment. In nature, the interfacial interactions between chitin and proteins are used for structural purposes and as a bio-cement, resulting in materials with properties unmatched by their man-made counterparts. Herein, we show that related supramolecular interactions can be harnessed to develop high strength green adhesives based on chitin nanocrystals (ChNCs), isolated from shrimp shells, and hen egg white lysozyme (HEWL) used in its monomeric or amyloid forms. Consolidation of the bicomponent suspensions, placed between glass substrates, results in long-range ordered superstructures. The formation of these structures is evaluated by surface energy considerations, followed by scanning electron, atomic force, and polarized microscopies of the consolidated materials. For $0.8 \mathrm{mg}$ of bio-adhesive (lysozyme, ChNCs or their composites), lap shear loads of over $300 \mathrm{~N}$ are reached. Such remarkable adhesion reaches maximum values at protein-to-ChNC ratios below 1:4, reflecting the synergy established between the components (ca. 25\% higher load compared to ChNCs, the strongest single component). We put the observed adhesive performance in perspective by comparing the lap-shear performance with current research on green supramolecular adhesives using natural biopolymers. The results are discussed in the context of current efforts to standardize the measurement of adhesive strength and bond preparation. The latter is key to formalizing the metrology and materials chemistry of bio-based adhesives. The proposed all-green system is expected to expand current developments in the design of bio-based adhesives.

\section{Introduction}

A range of synthetic materials with a short service-life yet a high persistence in the environment are responsible for a number of environmental hazards and the introduction of pollutants into the food chain. ${ }^{1,2}$ One class of materials that requires immediate attention is green adhesives with controlled interfacial strength. Beyond the development of adhesives, developing strength at interfaces from green building blocks has

\footnotetext{
${ }^{a}$ Department of Bioproducts and Biosystems, School of Chemical Engineering, Aalto University, P. O. Box 16300, FI-00076 AALTO, Finland

${ }^{b}$ Laboratory for Cellulose \& Wood Materials, Empa - Swiss Federal Laboratories for Materials Science and Technology, Überlandstrasse 129, 8600 Dübendorf, Switzerland ${ }^{c}$ Department of Health Science and Technology, ETH Zürich, 8092 Zürich, Switzerland ${ }^{d}$ Bioproducts Institute, Department of Chemical and Biological Engineering, Department of Chemistry and Department of Wood Science, University of British Columbia, 2360 East Mall, Vancouver, BC V6T 1Z4, Canada. E-mail: blaise.tardy@ aalto.fi; orlando.rojas@ubc.ca; gustav.nystroem@empa.ch

$\dagger$ Electronic supplementary information (ESI) available. See DOI: 10.1039/d1ta03215a
}

implications in nanocomposite and additive manufacturing. ${ }^{3-5}$ Therein, the properties of the materials formed are grounded on the interfacial supramolecular chemistry of the biocolloidal building blocks. The non-covalent and biodegradable aspects of these natural systems bear great promise for materials with an environmentally friendly end-of-life.

In insects and crustaceans, the principal interfaces used to develop mechanical resilience are those between proteins and chitins, which enable a rare combination of light weight, strength and toughness unmatched by modern synthetic systems. ${ }^{6-9}$ During the process of sclerotization, complex synergies develop between proteins and the surface of chitin nanofibers, essential to the high performance of their composites. ${ }^{\mathbf{1 0 , 1 1}}$ Chitin has also been found to be among the main components in the bio-cement of barnacle cyprids, i.e., the final larval stage and initial settling phase of barnacles, keeping such marine creatures well attached to underwater surfaces. ${ }^{12}$ Chitin was shown to be important not only for adhesion, but also for the structuring and self-assembly of the cement. In the case of adult barnacles, the cement has been 
shown to consist of self-assembled amyloid fibrils, which are fibrillary protein aggregates. ${ }^{13}$ Several studies have attempted to mimic cuticular structures using bio-based colloidal building blocks to achieve light-weight materials, mainly films, with high strength and toughness. ${ }^{14-16}$ In parallel efforts, proteinaceous building blocks have also been used to link exceptional mechanical properties with the added functional versatility arising from a vast possible combination of amino acid compositions. ${ }^{17,18}$ Herein, we combine chitinous building blocks readily obtained by deconstruction of biomass from waste streams ${ }^{19,20}$ with proteins, or their amyloids, to harness the functionality of natural bio-cement designs for the formation of sustainable adhesives.

We evaluate both individual chitin nanocrystals (ChNCs) and hen egg white lysozyme (HEWL) for their assembly and adhesion potential within a lap shear setup (Fig. 1). HEWL was chosen as a model protein as its aggregation into amyloids is well described. ${ }^{21,22}$ Under this confined evaporation induced self-assembly (C-EISA), adhesion strength characterization provides a robust framework for evaluating the structurefunction relationship of material assemblies of a wide composition range, including highly brittle composites that could not be evaluated otherwise. ${ }^{23}$ We first describe thoroughly the steps

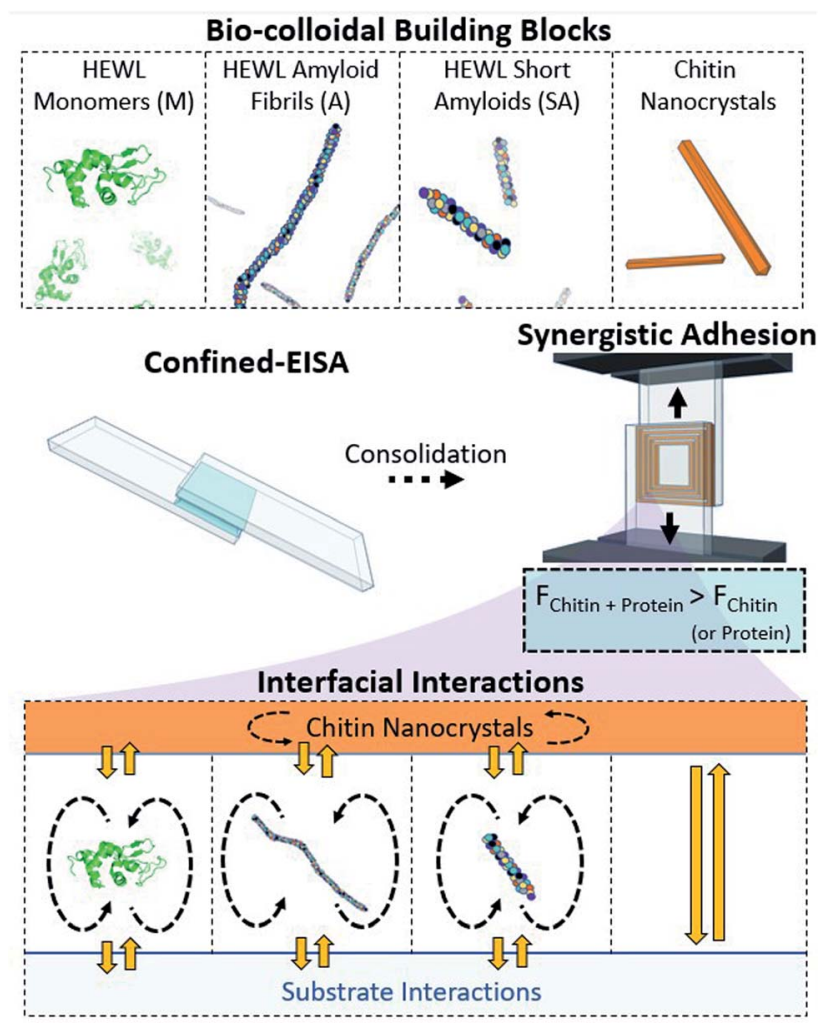

Fig. 1 Monomeric HEWL (M), HEWL amyloid fibrils (A), HEWL short amyloids (SA), and chitin nanocrystals (ChNCs) are herein used as structural adhesives produced by Confined-Evaporation Induced SelfAssembly (C-EISA) between glass slides. The combination of selfinteractions (dashed arrows), and heterogeneous interactions (yellow arrows) of the protein components and ChNCs is investigated within this framework. in isolating nanochitin from shrimp biomass, and identify the key factors in obtaining a "pure" nanochitin interface. Using model glass substrates, we demonstrate that the adhesion strengths of chitin and HEWL monomers (from $\mathrm{pH} 2$ suspensions) are comparable to that of cellulose nanocrystals (CNCs) formed at the same areal density. Surprisingly, the amyloid aggregates (both shortened and full-length) displayed a stark decrease in adhesion. Importantly, for amyloid : chitin mixing ratios of $1: 10$ and $1: 20$, a clear synergistic increase in adhesion strength is demonstrated, outperforming either of the mono-component systems. Importantly, this was related to the formation of long-range ordered superstructures between chitin and HEWL that maximizes the interactions at the colloidal interface in the consolidated composite (Fig. 1). These superstructures are described experimentally using polarized microscopy and scanning electron microscopy (SEM). Finally, we analyze the substrate-adherent interface (by SEM, atomic force microscopy (AFM) imaging, and force spectroscopy) to highlight the contribution of chitin and amyloids prior to failure of the (composite) adhesives. We conclude by placing the adhesive performance of these constructs in perspective with previously studied biopolymeric adhesives, with ChNC-amyloid composites displaying the highest specific strengths for the concentrations evaluated herein. The achievement of bionanocomposites as green adherents is an important step in the fabrication of advanced materials with a wide range of crossdisciplinary applications, and represents an important step towards the development of a functioning bioeconomy.

\section{Experimental}

\subsection{Chitin nanocrystal (ChNC) production}

2.1.1. Demineralization. $4 \mathrm{~L}$ of $\mathrm{HCl}(0.25 \mathrm{M})$ was added to $100 \mathrm{~g}$ of dried shrimp flakes (SIGMA). A magnetic stirrer was used to agitate the suspension at room temperature $\left(c a .20^{\circ} \mathrm{C}\right)$. The $\mathrm{pH}$ of the supernatant was monitored every $c a .1 \mathrm{~h}$, until a constant value of 1.1 was achieved (after $3 \mathrm{~h}$ ), indicating that the reaction was complete. ${ }^{24}$ The sample was subsequently added to a bag filter and washed with DI water until no changes in $\mathrm{pH}$ were observable upon further washing.

2.1.2. Deproteinization. The deproteinization was carried out by keeping the demineralized shrimp flakes in $4 \mathrm{~L}$ of $\mathrm{NaOH}$ ( $1 \mathrm{M}$ ) for $28 \mathrm{~h}$. The removal of proteins was followed by analyzing the supernatant by UV-vis at $280 \mathrm{~nm}$ (ref. 24) after 5 , 20.5, 24 and $28 \mathrm{~h}$ from the beginning of the process (Fig. S1 $\dagger$ ). The demineralized and deproteinized sample was again washed with DI water until a constant $\mathrm{pH}$ of $c a .8$ was reached.

2.1.3. Bleaching. Chlorine bleaching was carried out in a similar way as done for plant-based biomass. The demineralized and deproteinized samples were divided into four equal parts and put into polyethylene (PE) bags (ca. 20-25 g of chitin per bag). $200 \mathrm{~mL}$ of $\mathrm{pH} 4$ buffer was added to each bag, followed by the addition of $12.5 \mathrm{~mL}$ of sodium chlorite (stock solution consisting of $3.96 \mathrm{~g}$ dissolved in $50 \mathrm{~mL}$ of DI water) and the subsequent addition of DI water until the total added liquid content reached $500 \mathrm{~mL}$. The $\mathrm{pH}$ was measured and adjusted to $<5$ before closing the bags. Once closed, the bags were put into 
a water bath at $50{ }^{\circ} \mathrm{C}$ for $1 \mathrm{~h}$. To stop the reaction, $40 \mathrm{~g}$ of sodium sulfite was dissolved in $400 \mathrm{~mL}$ of DI water and then added to the bags. After being neutralized, the chitin was washed with DI water.

2.1.4. Homogenization. First, the excess water was removed from the coarse, washed chitin flakes. The concentration was then adjusted using DI water to $c a .3 \mathrm{wt} \%$ in a total volume of $300 \mathrm{~mL}$. At such concentration, the chitin was homogenized by using a T-25 Ultra-Turrax (IKA, Germany) at speed scale number 5 for two runs of 2 min each (with a $c a$. 1 min interval).

2.1.5. Hydrolysis. After the homogenization, part of the supernatant of the sedimented chitin suspension was removed and $37 \% \mathrm{HCl}$ ( $c a .12 \mathrm{M}$ ) was added, bringing the chitin suspension to a final $\mathrm{HCl}$ concentration of $3 \mathrm{M}$. By using a heated oil bath, the suspension was boiled under reflux and kept under such conditions for $2 \mathrm{~h}$. It took $c a .30 \mathrm{~min}$ for the suspension to reach reflux conditions. To stop the hydrolysis, the round bottom flask was removed from the oil bath and subsequently cooled in ice-cold water. Ice-cold DI water was also carefully added to the suspension, which was later washed twice (using an Eppendorf 5804 centrifuge at $4500 \mathrm{rpm}$ for $15 \mathrm{~min}$ ) and dialyzed against Milli-Q using a regenerated cellulose membrane with a cut-off size of 6-8 kDa.

2.1.6. Concentration adjustment and dispersion. The suspensions were tip-sonicated in batches of $200 \mathrm{~mL}$ for $3 \mathrm{~min}$ (2-1 s on-off cycles) at an amplitude of $30 \%$. A $2 \mathrm{~cm}$ diameter probe (Sonifier 450, Branson Ultrasonics Co.) was used, and an ice bath helped to avoid overheating of the sample. The concentration of the suspension was adjusted by evaporation at $50{ }^{\circ} \mathrm{C}$ inside a Teflon container with gentle magnetic stirring until a concentration of $c a$. 2.5 wt $\%$ was attained. A new tipsonication step was performed for $2 \mathrm{~min}$ at $40 \%$ amplitude (2-1 s on-off cycles). Centrifugation at $9000 \mathrm{rpm}$ for $10 \mathrm{~min}$ (with an additional 4 min of ramp up and 4 min ramp down) was carried out and the supernatant was kept, eliminating large particle aggregates possibly generated during the evaporation step. The concentration of the resulting suspension was finally adjusted to $2 \mathrm{wt} \%$ with Milli-Q water and the final $\mathrm{pH}$ was $c a .3$. The final ChNC samples were stored at $4{ }^{\circ} \mathrm{C}$ and were observed to be colloidally stable over several months.

\subsection{HEWL amyloid production}

HEWL amyloids were formed using a protocol based on incubation at low $\mathrm{pH}$ and high temperature, previously described by Lara et $a .^{21}$ Briefly, HEWL (Sigma-Aldrich) was suspended in Milli-Q to a concentration of $2 \mathrm{wt} \%$ and $\mathrm{pH}$ 2, adjusted using $\mathrm{HCl}$. The suspension was placed in a thermomixer (Eppendorf AG) at $90{ }^{\circ} \mathrm{C}$ and $400 \mathrm{rpm}$ for $24 \mathrm{~h}$. Short amyloids were prepared by subsequently tip-sonicating (Digital Sonifier 450, Branson Ultrasonics) the fully grown amyloid suspension for $20 \mathrm{~min}$ ( 2 second pulses) at $20 \%$ amplitude. ${ }^{25}$ Samples were stored at $4{ }^{\circ} \mathrm{C}$ prior to use.

\subsection{Lap preparation and lap shear test}

Suspensions of ChNCs (pH 3), monomeric HEWL (pH 2 adjusted with $\mathrm{HCl}$ ), HEWL amyloids ( $\mathrm{pH} 2$ ) and HEWL short amyloids ( $\mathrm{pH} 2$ ) were used at $2 \mathrm{wt} \%$ for preparing the laps. Mixtures of the protein monomers (M), or aggregates (amyloids (A) or short amyloids (SA)), with ChNCs were also prepared at 6 different composition ratios, i.e., $1: 20,1: 10,1: 4,1: 2,1: 1$ and $2: 1$. All suspensions were bath sonicated (Bandelin Sonorex Digitec) for $c a .1 \mathrm{~min}$. The laps were prepared by first casting $40 \mu \mathrm{L}$, at $2 \mathrm{wt} \%$, of the suspensions on one of the smooth sides of glass slides $(76 \times 26 \times 1 \mathrm{~mm}$; from VWR), and by subsequently covering a length of $c a .25 \mathrm{~mm}$ of such substrate with another glass slide (Fig. 1). All substrates were used as received. The upper substrates were supported in the free end by another glass slide of the same thickness during the confined evaporation induced self-assembly (C-EISA), as to allow the development of an even thickness across the lap during drying. All substrates had a frosted area covering one of their ends, which was used for increasing the friction with the clamp of the universal tensile testing machine (MTS-400, $2 \mathrm{kN}$ load cell) used for the lap shear test. All laps were dried under ambient conditions, at $c a .22{ }^{\circ} \mathrm{C}$ and $20 \%$ relative humidity. Although the bonds were apparently consolidated after a few hours ( $c a .4 \mathrm{~h}$ ), the lap shear tests were carried out after $c a .40 \mathrm{~h}$ after casting to ensure that all samples were fully dried. The lap shear tests were conducted at a strain rate of $1.5 \mathrm{~mm} \mathrm{~min}^{-1}$. All results were calculated based on at least four samples for each set of conditions.

\subsection{Imaging}

Prior to lap shear testing, all laps were photographed using a digital camera (16 MP resolution). Polarized optical microscopy was performed on selected samples using an Olympus SZX10 microscope, with the illumination, exposure and gain settings remaining unchanged across all images. A slight overexposure enabled the visualization of the fractures, while highlighting differences in birefringence patterns across the samples. Scanning electron microscopy images were recorded using a Zeiss Sigma VP field emission scanning electron microscope at $1.6 \mathrm{kV}$ and working distance of $c a .7 \mathrm{~mm}$. The monomeric HEWLs, their amyloids, and short amyloids were imaged by AFM (Bruker ICON3), with the samples being prepared from $\sim 0.05 \mathrm{mg} \mathrm{mL}^{-1}$ suspensions deposited on freshly cleaved mica substrates. The ChNCs were imaged by AFM using a JPK-Bruker NanoWizard IV XP on freshly cleaved mica. The length and width of the ChNCs were corrected for tip convolution according to previous work. ${ }^{26}$

\subsection{Atomic force microscopy (force mapping)}

The same procedure used to produce the lap shear specimens was used to prepare fractured bonds for AFM imaging. However, these samples were fractured out of plane after an additional drying step at $50{ }^{\circ} \mathrm{C}$ for at least $30 \mathrm{~min}$ to increase brittleness and result in clean fractures. Images of C-EISA samples were obtained using the QI-Nano mode on a Nanowizard 4 (Bruker, Germany) AFM using a cantilever with an $8 \mathrm{~nm}$ radius spherical tip (nominal spring constant of $40 \mathrm{~N} \mathrm{~m}^{-1}$ and a resonance frequency of $c a .260 \mathrm{kHz}$ ). The deflection was set at $10 \mathrm{nN}$, with $100 \mathrm{~nm}$ approach and retract distances. 


\subsection{Surface tension and contact angle measurements}

The surface tension was evaluated by using a Theta Flex optical tensiometer (Biolin Scientific) and the pendant drop method. The droplet volume was $12.5 \pm 0.5 \mu \mathrm{L}$ for all the suspensions. The reported values represent the average from at least 3 measurements, where the surface tension was automatically calculated from images of the droplet recorded over $1 \mathrm{~min}$, and at a frame rate of 1 image per second. Using the same instrument, contact angle measurements were performed by placing ca. $7 \mu \mathrm{L}$ droplets on the same glass substrates used for the CEISA. The droplets were also recorded over $1 \mathrm{~min}$, and at a frame rate of 1 image per second.

\section{Results and discussion}

\subsection{Building block preparation and self-interactions}

3.1.1. Chitin nanocrystals (ChNCs). Dried shrimp shells (SIGMA) were demineralized (mainly from $\mathrm{CaCO}_{3}$ ), deproteinized, bleached and hydrolyzed (Fig. 2a) to produce the ChNCs used for lap shear tests. Currently, this is the most commonly used and well-established method for the production of chitin. ${ }^{27}$ For sustainable large-scale production, however, biocatalysts and processing with green solvents (including ionic liquids) are being developed under the framework of future biorefineries..$^{20,28}$ In all cases, the use of closed-loop systems and the utilization of by-products are receiving great attention. The bench-scale demineralization and deproteinization methods used in this study allowed a suitable control of the process by monitoring the $\mathrm{pH}$ and light absorbance (UV-vis), ${ }^{24}$ respectively, to ensure pristine chitinous interfaces and minimum degradation. AFM characterization of the dried suspension indicated the successful production of nanocrystals having an average length of $262 \pm 122 \mathrm{~nm}$ and height of $8.7 \pm 3.8 \mathrm{~nm}$ (Fig. 2b and c). The latter was calculated ${ }^{26}$ to an equivalent circular diameter of $17 \mathrm{~nm}$. The ChNCs were well-dispersed and presented a segmented morphology along the principal axis, although a high severity hydrolysis was used herein. Particle purity and integrity as well as colloidal stability are important to ensure proper packing, alignment, and inter-particle/interfacial contact. These are crucial when evaluating self-interactions

a) ChNC Production

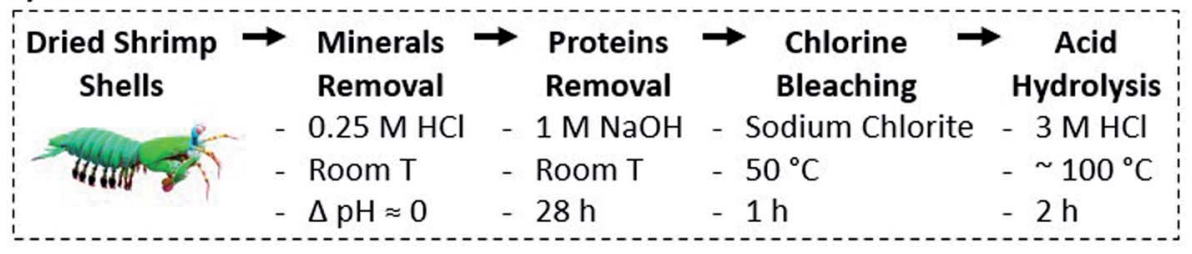

b) ChNC

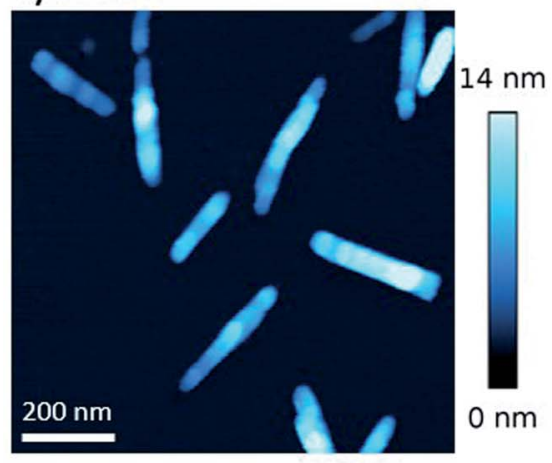

d) ChNC Assemblies c) ChNC Size Characterization

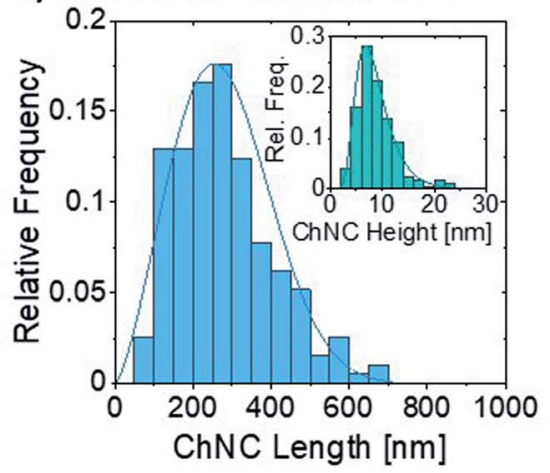

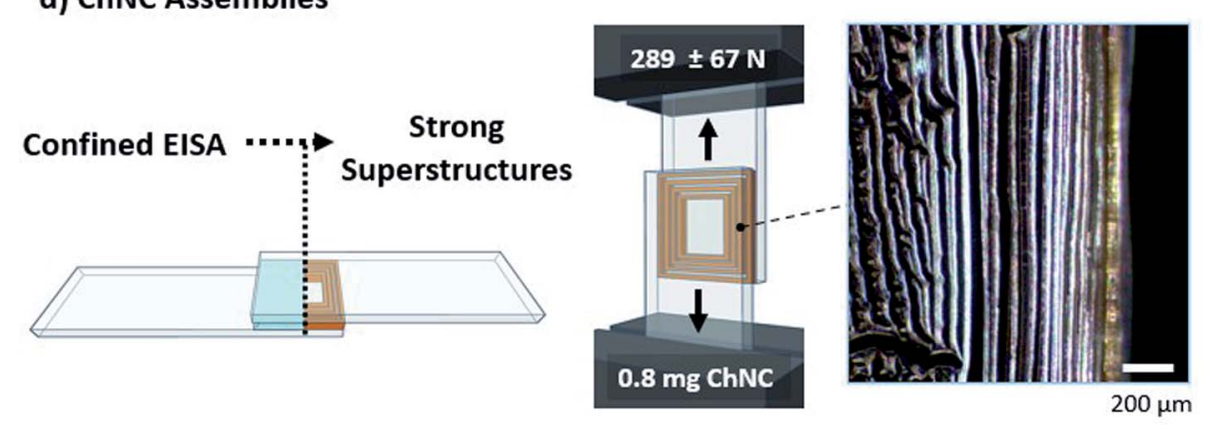

Fig. 2 (a) Chitin nanocrystal (ChNC) isolation route. (b) Resulting ChNC morphology as shown by the AFM height image. (c) Histograms of the resulting ChNC dimensions measured from AFM images. (d) Schematic representations of the bond formation and lap shear test. The inset shows the long-range order of the lamellar structure, imaged through polarized optical microscopy. Both filters are oriented at $45^{\circ}$ with respect to the vertical orientation of the image. 
and the synergy between ChNCs and proteins. For practical applications, however, a high level of purity may not be required and a proper balance between purification protocol, cost, and performance should be established.

The nanocrystals were self-assembled by drying $40 \mu \mathrm{L}$ of the $2 \mathrm{wt} \%$ suspension between glass microscopy slides (Fig. 2d), to a final areal density of $c a .0 .13 \mathrm{mg}$ ChNCs per $\mathrm{cm}^{2}$ and a total of $0.8 \mathrm{mg}$ ChNCs per bond. As observed from polarized optical microscopy, such confined evaporation induced self-assembly (C-EISA) of the suspension resulted in the formation of birefringent lamellae, with ChNCs aligned parallel to the lamellae orientation (Fig. 2d and S2a†). Similar to our previous observations on CNCs, the formation of such superstructures can be attributed to capillary forces driving the confined self-assembly process. $^{23}$ The controlled evaporation process promoted the formation of strong supramolecular interactions within the lamellae and between the biopolymeric colloids and substrate. Consequently, $0.8 \mathrm{mg}$ of ChNC assemblies had a high lap shear strength (Fig. 2d), failing at ca. $289 \pm 67 \mathrm{~N}$, or $1.45 \pm 0.4 \mathrm{MPa}$. Compared to CNCs previously tested using a similar setup, ChNCs reached roughly equivalent ultimate loads, as $1.1 \mathrm{mg}$ of CNCs at $0.41 \mathrm{mg} \mathrm{cm}^{-2}$ failed at $269 \pm 99 \mathrm{~N}^{23}$ A more detailed comparison with recent biocolloidal adhesive performance is herein presented in Section 3.4.

Additionally, 1 and $4 \mathrm{wt} \%$ suspensions (equivalent to areal densities of 0.065 and $0.26 \mathrm{mg}$ ChNCs per $\mathrm{cm}^{2}$ ) were also tested and found to result in strong assemblies (Fig. S3†). The more diluted suspension failed at an average ultimate load of $236 \pm$ $44 \mathrm{~N}$, while the more concentrated one failed at $496 \pm 75 \mathrm{~N}$. Similarly to the observations made for cellulose nanocrystals, ${ }^{23}$ the relationship between areal density and strength was not linear. Furthermore, the impacts of solution volume and concentration are expected to affect the areal density and the time before gelation happens, provided homogeneous wetting occurs within the curing bond. ${ }^{23}$ As substrate failure was occasionally observed at $4 \mathrm{wt} \%$, an optimal concentration of $2 \mathrm{wt} \%$ was used for further analysis with the protein components and the mixtures.

3.1.2. Monomeric and aggregated HEWL. Protein-based colloidal building blocks were also prepared and analyzed, as inspired by the strong interactions naturally occurring e.g. in mussels byssus and barnacle cement, where proteins, also in self-assembled states, strongly attach to hard substrates. ${ }^{12,13}$ In short, HEWL amyloid fibrils were produced by hydrolyzing the HEWL at $90{ }^{\circ} \mathrm{C}(\mathrm{pH} 2)$ for $24 \mathrm{~h}$ (Fig. 3(a1)). During incubation, hydrolyzed peptide fragments aggregated into amyloid fibrils of several microns by ca. 4-10 nm (Fig. 3(a2)). ${ }^{21,25}$ Short amyloids, ca. $75 \mathrm{~nm}$ by $6 \mathrm{~nm}$, were also produced by tip sonicating the amyloid fibrils as previously reported (Fig. 3(a3)). ${ }^{25}$ Monomeric HEWL (M), amyloid fibrils (A), and short amyloids (SA) were all used to produce lap shear specimens through the C-EISA of 40 $\mu \mathrm{L}$ of individual suspensions ( $2 \mathrm{wt} \%$ and $\mathrm{pH} 2)$.
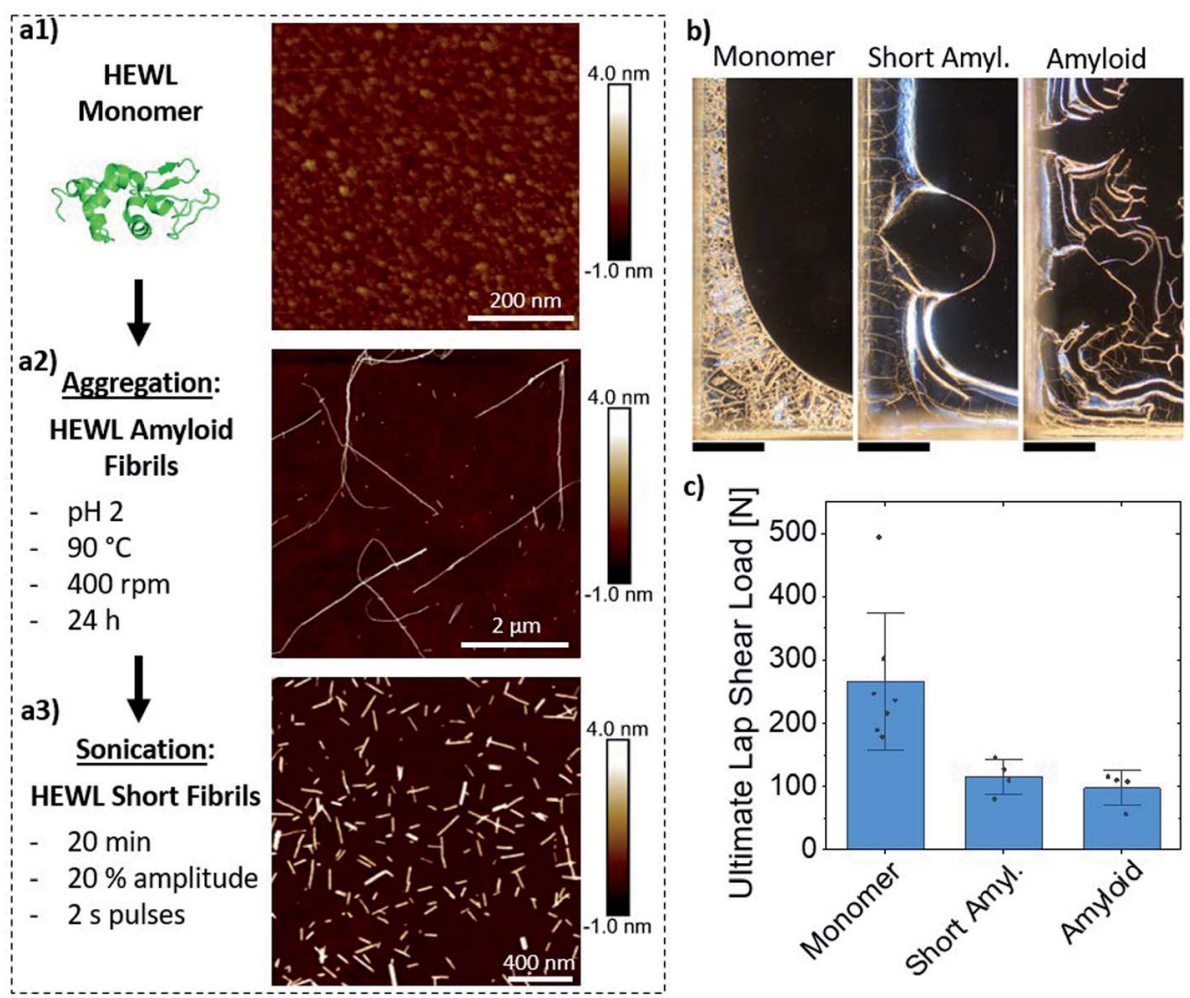

Fig. 3 HEWL amyloid fibril formation process and AFM images illustrating the morphology of the (a1) monomeric HEWL, (a2) HEWL amyloid fibrils, and (a3) short fibrils. (b) Polarized optical microscopy images of the edge of laps formed by C-EISA of HEWL in the various aggregation states, showcasing the reduced formation of lamellar structures near, and parallel to, the edges of the bond as compared to ChNCs (Fig. 2d). Scale bars are $400 \mu \mathrm{m}$. The samples were slightly overexposed due to low birefringence. (c) Lap shear test comparing the mechanical strength of the protein-based assemblies. 
All three suspensions formed organized structures upon drying as evidenced by birefringent patterns under crosspolarized illumination (Fig. 3b). Their morphology, however, was substantially different from those formed by ChNCs, with reduced formation of well-oriented structures near the edges. Nevertheless, the monomeric HEWL resulted in similar lap shear strength when compared to ChNCs (Fig. 3c). Interestingly, amyloid fibrils and short fibrils resulted in weaker adhesion than their monomeric counterparts, with ultimate loads approximately $60 \%$ lower compared to ChNCs and monomeric HEWL. We hypothesize that this difference in strength between the protein assemblies may have occurred because of a more favorable and organized distribution of interactions (mainly hydrogen bonding) along the consolidated materials formed from monomeric suspensions. Note, for instance, that the monomers assembled into highly ordered microcrystalline domains of at least two different conformations, i.e., needle- and plate-like crystals, as observed in Fig. S4. $\dagger$ Such range of conformations in the dried material may have also contributed to the large variation in lap shear strength. In contrast, no such microcrystalline domains were observed in the consolidated amyloid adhesives, as suggested by the low birefringence from these samples (Fig. 3b).
3.1.3. Synergy between ChNCs and HEWL. We further evaluated the mechanical performance of ChNC-protein adhesive compositions at six different ratios and compared them to the single components previously tested. ChNCs were mixed with either monomeric HEWL (M), HEWL amyloids (A) or short amyloids (SA) at protein : ChNC ratios ranging from $1: 20$ to $2: 1$. The suspensions were used to produce the lap shear specimens at the same areal density $\left(0.13 \pm 0.01 \mathrm{mg}\right.$ per $\left.\mathrm{cm}^{2}\right)$ and overlap area used for single component adhesives. Due to positive charges in all components involved, no aggregation was observed in the dispersed state. This contrasts with mixtures between nanocelluloses and proteins, where e.g. TEMPOoxidized cellulose nanofibrils and HEWL undergo electrostatic complexation. ${ }^{22}$ Furthermore, to highlight the ease of a lap shear approach for a systematic mechanical characterization, suspensions at different mixing ratios of amyloids and ChNCs were deposited on polypropylene substrates and subjected to EISA to form films instead of laps. For such non-confined framework, amyloid fibrils alone resulted in highly fractured films well adhered to the non-polar substrate, while easily detachable and cohesive films occurred upon addition of increasing amounts of chitin (50\% or more) (Fig. S5 $\dagger$ ).
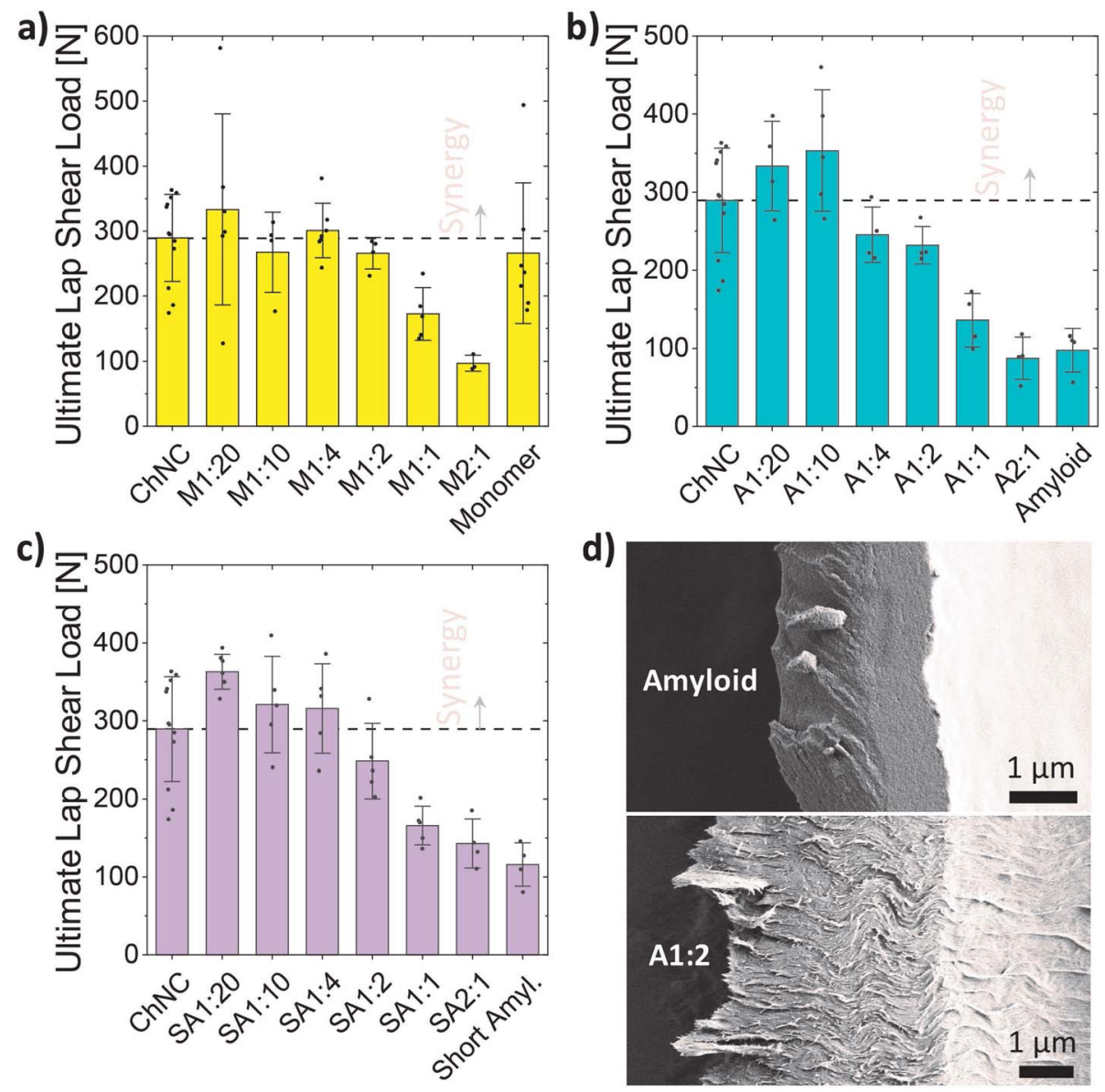

Fig. 4 Ultimate load for the lap shear test of bonds formed from suspensions of varying mixing ratios of ChNCs and (a) monomeric HEWL (N), (b) HEWL amyloids (A), or (c) short amyloids (SA). (d) SEM images of the surface of a fractured lamella after failing during lap shear tests. Top, only HEWL amyloids. Bottom, A1 : 2 sample (shear lips are seen protruding away from the left side of the lamella, indicating higher plasticity than that of HEWL amyloids alone). 
Interestingly, we observed that the drying time for such films containing high loadings of chitin was substantially longer (up to $30-50 \%$ ), potentially due to a higher amphiphilicity of the proteins, allowing faster dewatering.

The ultimate loads resulting from lap shear tests pointed to a synergistic interaction between ChNCs and all HEWL states evaluated herein (Fig. 4a-c). At a 1:20 ratio, the maximum loads for short amyloids, long amyloids, and monomeric HEWL were 25,15 , and $15 \%$ higher than that of individual chitin, respectively, which itself was higher than that of any of the protein systems evaluated (although close to the monomeric HEWL adhesion strength). Different from short amyloids and monomeric HEWL, the maximum strength for amyloid-ChNC composites was observed at a $1: 10$ ratio, and was $22 \%$ higher than that of ChNCs. Such a slightly lower synergy when compared to short amyloids was potentially a result of the larger average size of the protein component, which may more easily disrupt the order of the tightly packed ChNC assemblies. We also observed that the synergy at $1: 10 \mathrm{ChNCs}$ to amyloids, was not maintained when casting $4 \mathrm{wt} \%$ suspensions (Fig. S3 $\dagger$ ). This was likely caused by the higher charge density ${ }^{25}$ of the amyloids or by their slower diffusion relative to ChNCs. Either one of these effects, or the combination of both, may have led to an

a)

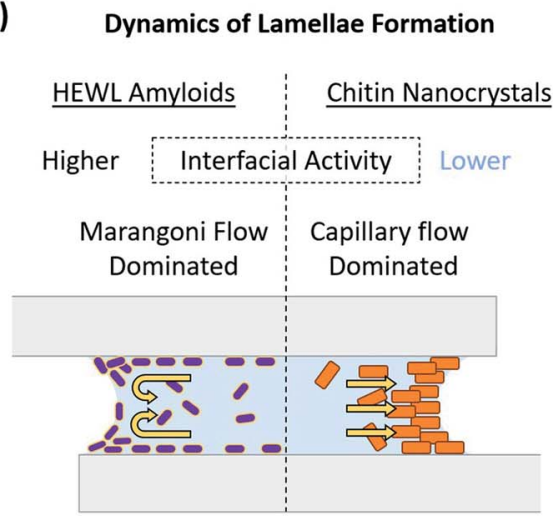

c) HEWL Amyloids

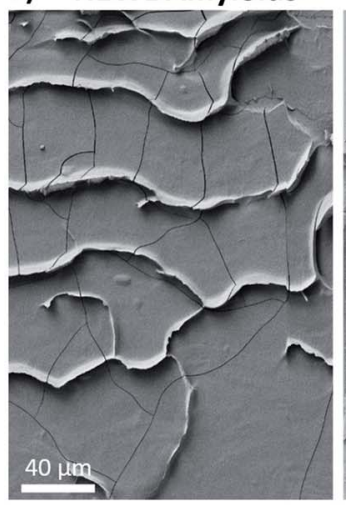

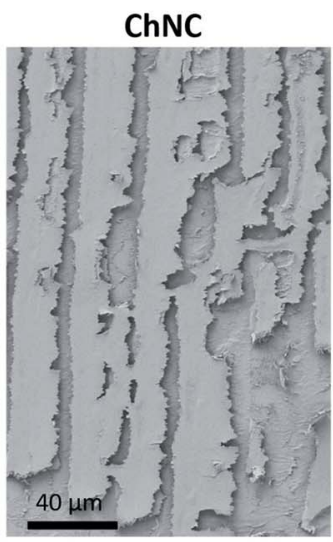
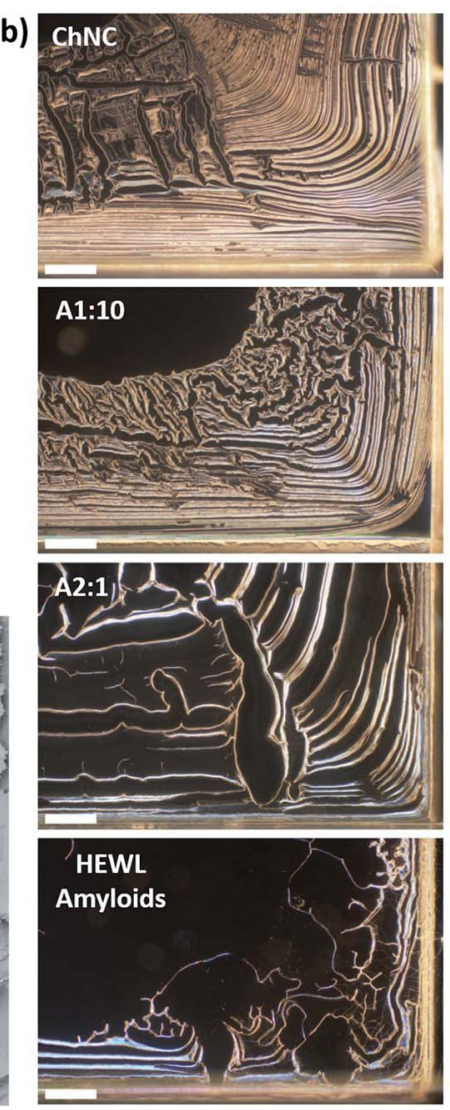

Fig. 5 (a) Factors associated with lamellae formation and particle distribution along the bond. (b) Polarized optical microscopy of ChNCs, HEWL amyloid fibrils, and their mixtures at $1: 10$ and $2: 1$ (amyloid : ChNC) ratios. Scale bars represent $400 \mu \mathrm{m}$. (c) SEM images of the edge region of a fractured bond formed by amyloids (left) and ChNCs (right). These bonds were fractured in the out-of-plane direction. 
amyloid assemblies evidenced the occurrence of brittle fractures, mostly characterized by a smooth fracture surface of the lamellae (Fig. 4d, top). On the other hand, increasing amounts of ChNCs resulted in relatively higher plasticity of the bonds, as shown by a rough fracture surface and by shear lips protruding away from the lamellae (Fig. 4d, bottom). Differences in the area connecting both glass substrates, however, might have affected the strength of the bonds.

\subsection{Lamellae formation and load-bearing area}

We further investigated the formation of the lamellae and the contact areas for the bonds formed using HEWL amyloids or their mixtures with ChNCs, and linked these two factors to the loadbearing ability of the self-assembled materials. Thereafter, ChNCs and amyloids were the main focus due to the regularity of the self-assembled structures and their high synergy.

On a macro scale, a clear increase in the areal coverage of the central part of the bond could be observed for the mixtures having increasing amounts of amyloids (Fig. S6†). At the same time, those bonds containing larger quantities of ChNCs presented a more organized lamellar structure, preferentially located near and parallel to the edges (Fig. $5 \mathrm{~b}$ and c). This trend can be explained by the wetting characteristics of the suspensions and their surface activity, which affect the receding motion of the solid-liquid contact line of the suspensions as well as the particle flow during drying.

The increased surface activity of the amyloids, compared to ChNCs, lowered the surface tension of the suspensions while maintaining a nearly unchanged contact angle across the different ratios (Table 1). With these quantities, the work of adhesion $\left(W_{\mathrm{SL}}\right)$ of the suspensions to the substrates could be estimated using the Young-Dupré equation $\left(W_{\mathrm{SL}}=\gamma_{\mathrm{L}}(1+\right.$ $\cos \Theta)$ ), where $\gamma_{L}$ is the surface tension and $\Theta$ is the contact angle. When compared to ChNCs, the amyloid suspension presented a $21 \%$ lower work of adhesion to glass, while at the synergistic ratio of $1: 10$, the reduction was in the order of $11 \%$ only. Although this equation is usually applied for pure liquids, in the context of this work it should provide a qualitative comparison of how the adsorption of particles at the air-liquid and solid-liquid interfaces affects the water interaction with the substrate. The work of adhesion, for instance, is known to be proportional to the depinning force of the three-phase contact line during drying. ${ }^{29}$ Therefore, the decreased work of adhesion for amyloid-rich suspensions may have facilitated the depinning of the contact line, thus modifying the particle distribution across the bond.

Together with such higher mobility of the interface for the suspensions containing amyloid particles, Marangoni flow ${ }^{30,31}$ and particle induced drying-front instabilities ${ }^{32}$ might have also played a key role in particle distribution towards the central areas of the bond. When Marangoni flow occurs, a fraction of the particles are known to accumulate near the three-phase contact line (near the substrate), while others immediately above it are repelled from the interface by Marangoni eddies (Fig. 5a). ${ }^{30,31}$ These instabilities near the air-liquid interface maintain a high and locally inhomogeneous concentration of particles along the eddy region. When depinning occurs, multibranched lamellar patterns are formed (Fig. 5b and S6 $\dagger$ ). ${ }^{30}$ Capillary flow, on the other hand, is the main mechanism

Table 1 Comparison of the surface tension, contact angle to glass, and consequent work of adhesion of suspensions of ChNCs, HEWL amyloid fibrils, and their mixture at $1: 10$ (amyloid : ChNC) to glass

\begin{tabular}{llll}
\hline & ChNC & A1 to 10 & Amyloid \\
\hline Surface tension $\left[\mathbf{m J ~ m}^{-2}\right]$ & $71.7 \pm 0.2$ & $63 \pm 0.5$ & $56.7 \pm 0.5$ \\
CA $\left[^{\circ}\right]$ & $20 \pm 0.8$ & $17 \pm 0.3$ & $20.5 \pm 3.5$ \\
Work of adhesion of suspensions $\left[\mathrm{mJ} \mathrm{m}^{-2}\right]$ & 139.1 & 123.3 & 109.7 \\
Normalized work of adhesion of & 1 & 0.89 & 0.79
\end{tabular}

suspensions
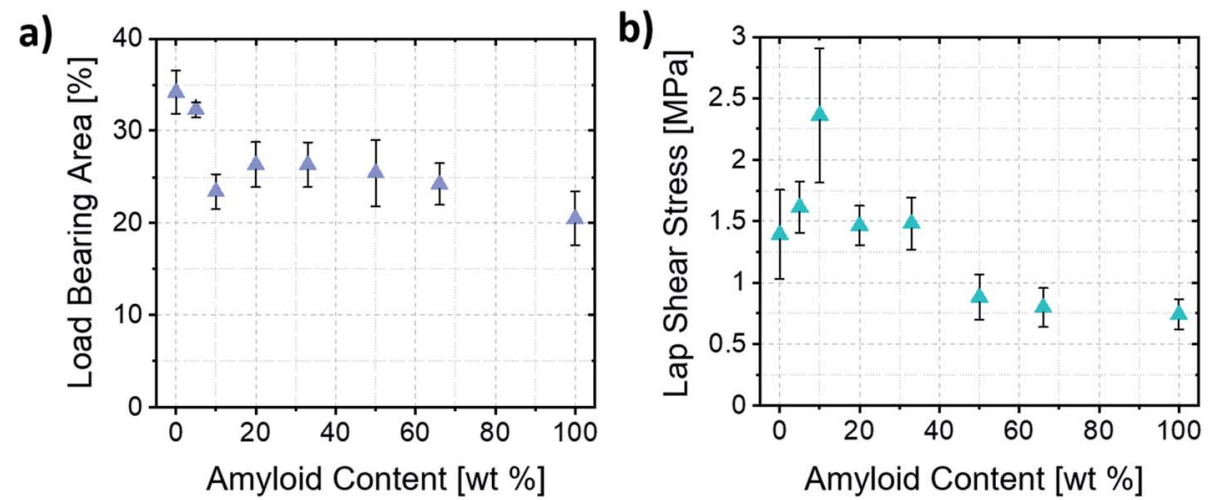

Fig. 6 (a) Load-bearing area estimated based on image analysis of the lamellae area. (b) Ultimate lap shear stress of the amyloid/ChNC assemblies at different mixing ratios. 
responsible for the accumulation of particles near the threephase contact line for ChNC-rich suspensions. Such flow is commonly attributed as the main cause for the "coffee-ring" effect usually seen in non- or weakly surface active particles in suspension, e.g., for CNC suspensions. ${ }^{33,34}$

Despite the complexity of the multiple dynamic events occurring simultaneously in such systems, we observe from the mechanical tests that the highest strengths may have occurred when capillary flow of particles towards the edges dominates the inward flow of particles. Additionally, a high work of adhesion of the dispersion may promote the formation of more organized and compact lamellar structures. Under such conditions, instead of having the amyloids forming a film covering the glass, they mostly contribute to the strength of the load bearing lamellae. The observed synergisms, however, cannot be explained solely by such assembly considerations, with the interfacial interactions between the building blocks after consolidation (further discussed in Section 3.3) also having fundamental importance.

Clearly highlighting such mechanism of formation of the lamellae allowed us to estimate the load-bearing area of the bonds and the resulting stresses upon shear failure. The thinner lamellae and the formation of films that do not bridge both substrates (Fig. 5c, left) resulted in samples having smaller load-bearing areas when higher ratios of amyloid fibrils were used. Importantly, this shows that the macroscopic area covered by the self-assembled material did not necessarily correlate with the load-bearing area, and that mostly the lamellae were responsible for transferring the load between substrates. Therefore, with such consideration, only the area covered by the lamellae was considered for the estimation of the

a) AFM of the Lamellae

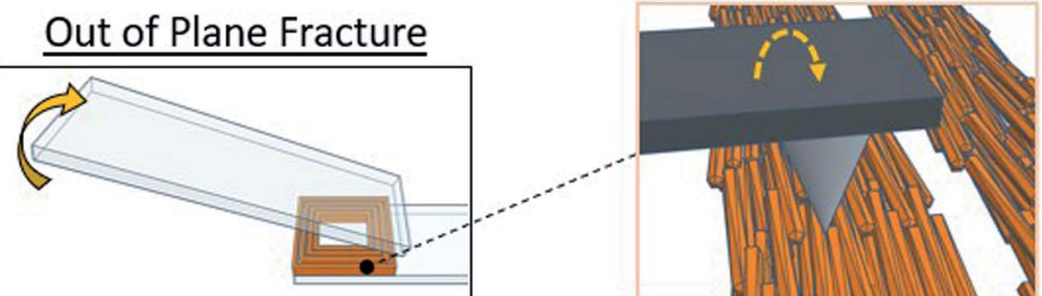

b) HEWL Amyloid

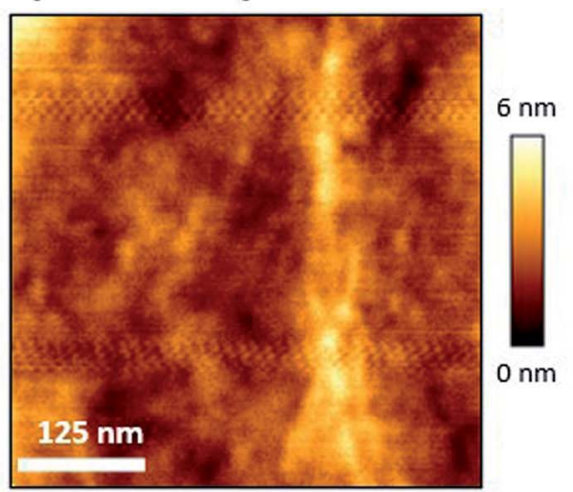

d) A1:10

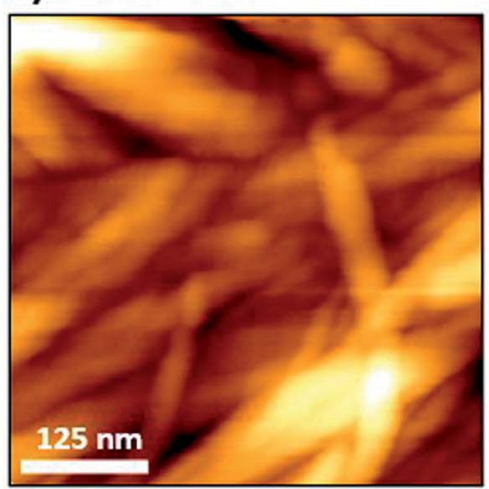

c) A2:1

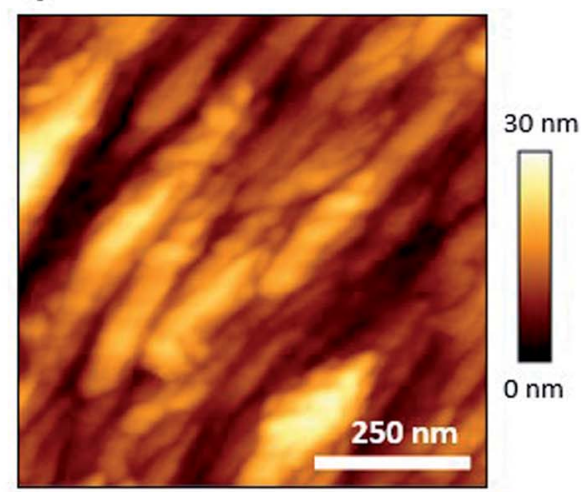

e) $\mathrm{ChNC}$

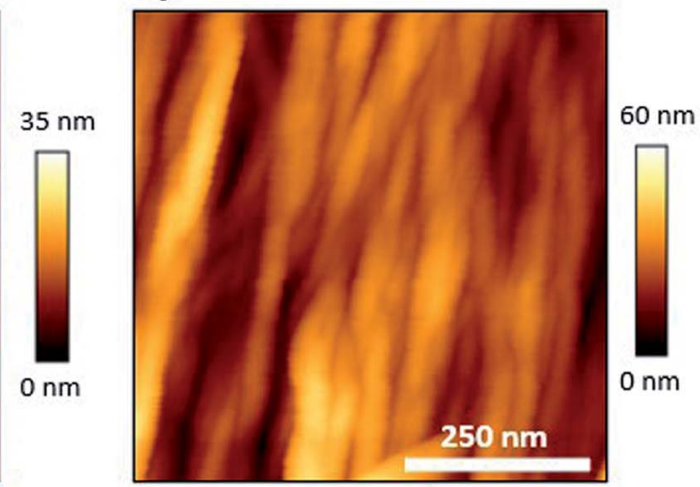

Fig. 7 (a) Schematics representing the out-of-plane fracturing of the bond, which was used for reducing plastic deformation. AFM height images of the lamellae of (b) HEWL amyloids, and their mixtures with ChNCs at (c) $2: 1$, (d) $1: 10$, and (e) $0: 1$ ratios. A 3D map of images (c) and (e) can be observed in Fig. S8. $\dagger$ 
load-bearing area and ultimate stress acting upon the assemblies (Fig. 6a and b).

The ultimate shear stresses followed the same trend as the ultimate load. The synergy, however, was even more evident for samples containing ca. $10 \mathrm{wt} \%$ amyloids (A1 : 10). Such result shows the importance of the wide, well-ordered lamellae (Fig. S2a and $\mathrm{b}^{\dagger}$ ) occurring near the edges of the bond for the load-bearing capacity of the assemblies. Furthermore, the different mechanisms of formation of the lamellae explained earlier may also result in more disordered assemblies within the lamellae when the Marangoni flow is the main driver of lamellae formation. Such a hypothesis is corroborated by the fact that an increased order and alignment is known to increase the mechanical properties of anisotropic particle assemblies. ${ }^{35}$

\subsection{Atomic force microscopy (AFM) analysis of lamellae}

Intimate contact between natural biopolymers, as well as biopolymers and substrate, will define much of the ability of adhesive interactions to occur. AFM characterization was used to further investigate such interfaces, e.g. as associated with the increased adhesion strength at a $1: 10$ mixing ratio. Lap shear samples were rapidly fractured out of plane, with the objective of preserving the structure of the lamellae with minimum plastic deformation (Fig. 7a). ${ }^{23}$

From a structural point of view, amyloids resulted in very compact assemblies, as shown by the low roughness of the fractured surface when compared to the ChNC-containing bonds (Fig. 7b and c). Difficulties in identifying individual amyloid fibrils may be a result of the aggregation of residual peptide fragments in the amyloid suspension upon drying, forming a smoother surface. Convolution effects of the AFM tip may have also compromised the visualization of individual fibers. Furthermore, chitin bonds showed a high alignment of the ChNCs at the interface (Fig. 7e), as was observed for CNCs. ${ }^{23}$ This alignment was also clearly observed for the amyloid : ChNC $2: 1$ mixing ratio, with the amyloid fibrils in intimate contact with the ChNCs. Some amyloids crossing ChNCs could be distinguished (Fig. 7c). For the 1: 10 ratio, however, the alignment was apparently less pronounced (Fig. 7d). This is possibly a result of higher adhesion in the out-of-plane loading direction, and of higher ductility of the assembly. Nevertheless, as evidenced by polarized microscopy for these three ChNCcontaining samples, all the mixtures presented a high birefringence (Fig. S2 and S7†), confirming the alignment of the ChNCs within the structured area of the biocolloidal assemblies.

When comparing the adhesion energy of the AFM tip to different lamellae, it was evident that ChNCs presented a lower interaction with the $\mathrm{OH}$ terminated tip (Fig. 8a-d). Importantly, the highest maximum force of adhesion occurred for the amyloid-rich samples, while a more heterogeneous distribution of such adhesion force occurred for the sample containing $c a$.
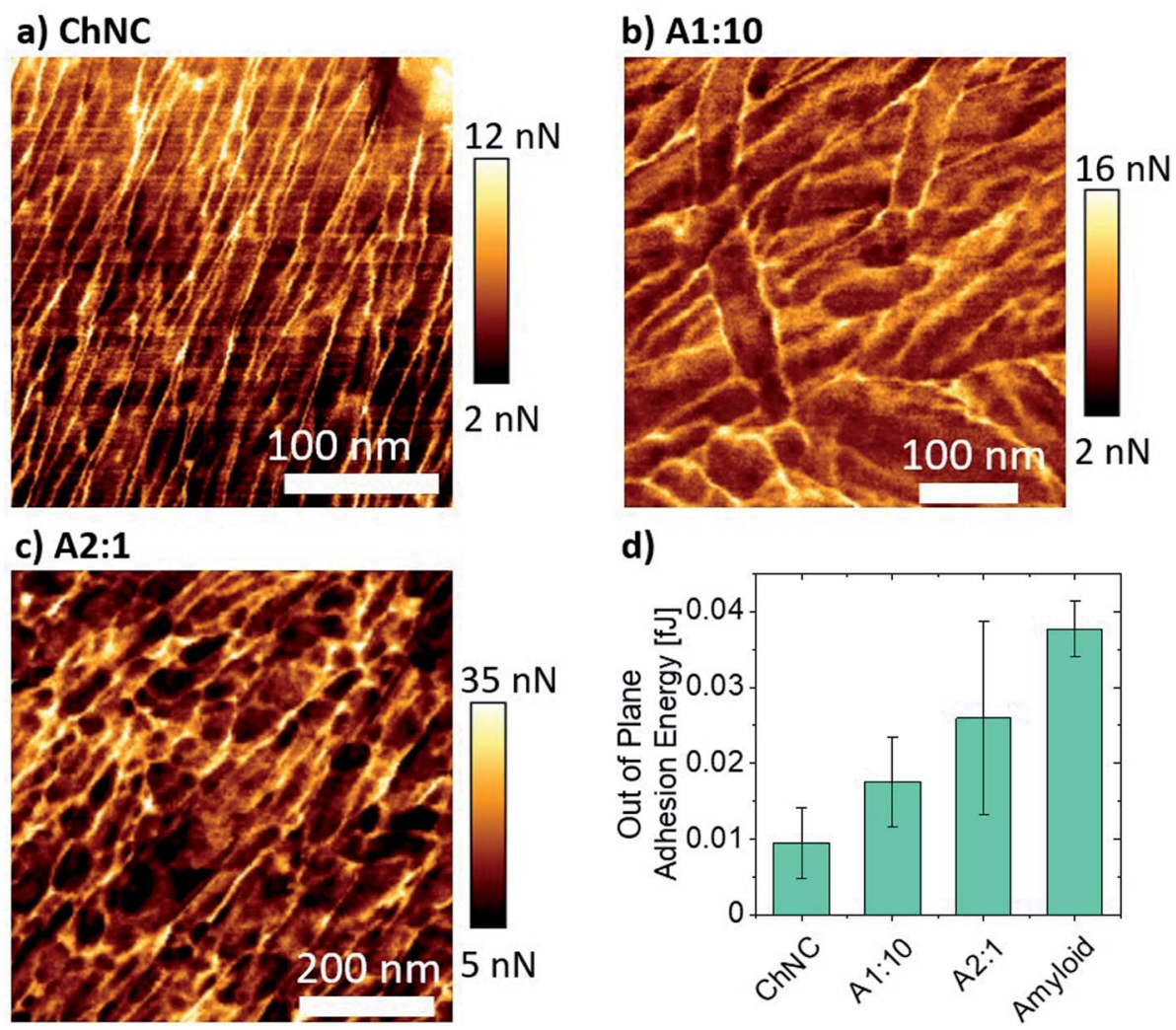

d)

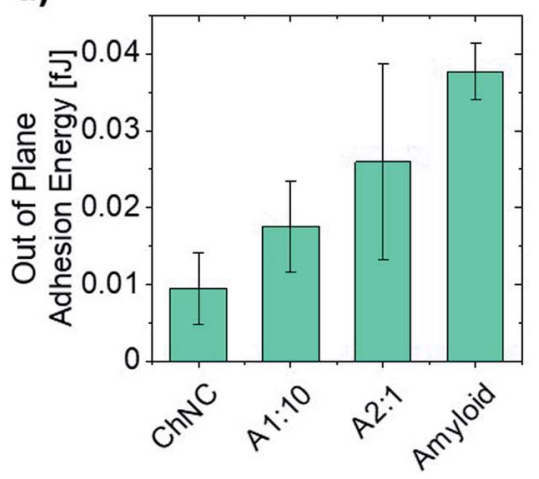

Fig. 8 Mapping of the maximum force of adhesion for (a) ChNCs, (b) $1: 10$, and (c) $2: 1$ ratios of amyloids to ChNCs. (d) Adhesion energy of the AFM tip to the lamellae represented in (a), (b), and (c) force maps. The average adhesion energy was calculated from the area under the AFM retraction force curves composing a force map image. 
$66 \mathrm{wt} \%$ amyloids (A2:1) as compared to the more ChNC-rich A1 : 10 sample (Fig. $8 \mathrm{~b}-\mathrm{d}$ and S8 $\dagger$ ). Such observations may imply that, although amyloids may increase cohesion for the lamellae due to high supramolecular interactions, a more heterogeneous distribution of amyloids may impede proper stress transfer across the material. Also, high supramolecular interactions may result in regions of more brittle nature, making the lamellae more susceptible to catastrophic failure once fractures are generated within the lamellae. In a biological context, similar observations may occur; although the crosslinking of the components and hydration effects may play a crucial role in determining the composition of highest possible synergy between chitin and proteins for a given marine organism, it is important that both components are organized and homogeneously distributed within the materials.

\subsection{Outlook on biopolymeric and biocolloidal adhesives}

Supramolecularly bound systems, as encountered in most natural structures, present the unique characteristics of enabling directional strength combined with reversible interactions. Furthermore, the absence of a highly cross-linked network (such as that present in epoxy glues), allows for the penetration of enzymes and thus the eventual biodegradation of the supramolecular adhesive matrix. Nevertheless, direct comparison across different studies on the interfacial supramolecular interactions of biopolymeric systems and the resulting adhesion strength remain challenging and should be done with care, as several variables need to be cautiously considered, such as contact area, long-range order, etc. To this end, we have quantitatively compared our results on ChNCs and HEWL with recent literature on CNCs and lignin nanoparticle-based adhesive systems (Fig. 9), where similar solid concentrations, adhesive mass per unit area, and substrates allow a direct comparison.

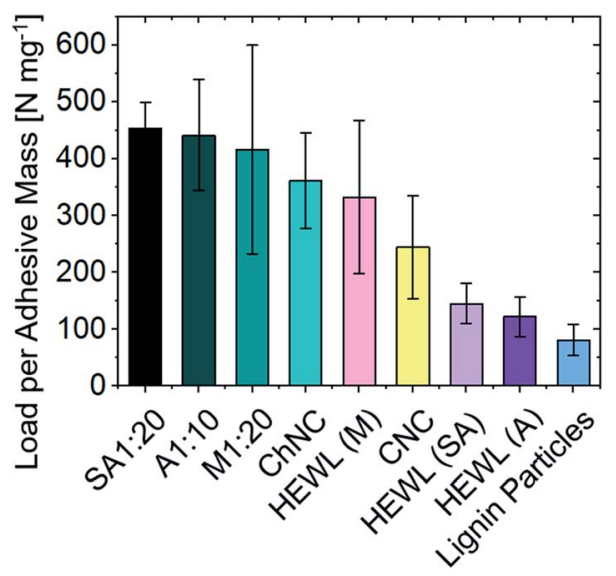

Fig. 9 Comparison of the specific ultimate strength between biocolloid-based adhesives. The substrate material (glass) and areal densities are kept nearly constant across the studies (from 0.13 to $0.18 \mathrm{mg} \mathrm{cm}^{-2}$ ). Lignin nanoparticle ${ }^{38}$ and $\mathrm{CNC}^{23}$ values are extracted from recent literature. A detailed comparison of the values herein showcased is available in Table S1. $\dagger$
For samples of similar areal densities (from 0.13 to $0.18 \mathrm{mg}$ $\mathrm{cm}^{-2}$ ), the composite assemblies between ChNCs and proteins obtained herein present the highest interfacial interactions, followed by CNCs, ChNCs, monomeric HEWL, amyloids, and finally by lignin nanoparticles. The lower performance of lignins may be associated with their amphiphilic nature as well as the inherent packing limitations of spherical particles, both detrimental to the development of strong interfacial interactions (the maximum random packing density of spheres is $c a$. 0.64 and that of spherocylinders is $c a .0 .72) .{ }^{36}$ The low selfcohesion of lignin, although not ideal for supramolecular adhesion, can be overcome via covalent cross-linking, leading to high strength adhesives. ${ }^{37}$ Conversely, the higher strengths observed for CNCs and ChNCs may have been enabled by good packing of the rod-like particles (resulting in higher contact area between colloids), by extensive hydrogen bonding, and also by the strong nature of the crystalline building blocks themselves. Furthermore, the crystallization of monomeric HEWL (Fig. S4 $\dagger$ ) may have contributed to the formation of strong bonds.

The current literature on biopolymeric adhesives exploiting supramolecular interactions is not grounded in formalized experimental practices, making quantitative comparison challenging. For instance, substrate roughness and its (surface) chemical composition are not frequently reported or standardized across studies. Typically, biopolymeric adhesives are explored with wood as a substrate, where surface roughness, porosity, and surface treatment significantly affect adhesion strength and prevent normalization of the stresses to the actual contact area. Other considerations may include age of the wood (young $v s$. old growth), location of harvest, drying treatments, etc. For instance, $c a$. 30 to $35 \%$ variation in ultimate adhesion strength is reported for gelatin on bamboo or maple, and for casein on beech or spruce substrates. ${ }^{39,40}$ Furthermore, unspecified contact area or missing information on other preparation procedures (temperature, humidity, pressure, etc.) also hinders direct comparisons between biopolymeric adhesive systems. Therefore, only qualitative insights should be extracted. For instance, when considering bovine serum albumin as a non-crosslinked biopolymeric adhesive, the impact of substrate chemistry substantially affected adhesion strength, where glass showed a lap shear adhesion 8.5 times stronger than that of PMMA and PC. ${ }^{41}$ Among other important variables, this is likely due to the wetting phenomenon, as also described herein, that can affect the intimate contact between biopolymers and substrates as well as the overall packing of the adherent.

Additionally, other qualitative information can be extrapolated based on optimized adhesive strengths for each class of material on a similar substrate. For instance, when comparing a range of biopolymeric adhesives used for wood adhesion, the highest lap shear strengths are achieved from protein systems, followed by starch, cellulose and chitosan, and finally from hemicelluloses and similar structures such as alginate (Fig. S10†). This suggests that proteins could present higher adhesive performances, although polysaccharides may be preferred due to their considerably higher available volume, 
and considering the valorisation of wastes. For commercial applications, other important factors should be considered, including biomass sourcing, fractionation methods, and purity. They not only affect the structure and performance, ${ }^{27}$ but also the technoeconomic viability of any practical endeavor. Importantly, the addition of biocolloidal nanofillers to adhesive systems can markedly enhance the adhesion strength (e.g. when using starch or polyvinyl acetate (PVAc)-based adhesives). For instance, improvements between $c a$. 35 and $75 \%$ are observed when using CNFs, ${ }^{42}$ and up to $100 \%$ when using CNCs. ${ }^{43}$ Furthermore, studies on the improvement of biopolymeric adhesives through synergisms across different classes of biopolymers and biocolloids, such as in the present study, are scarce. However, the supramolecular chemistry of such building blocks bears great potential for the development of high-performing, sustainable structural adhesives.

\section{Conclusion}

Chitin nanocrystals (ChNCs) developed a high shear-adhesion following the same mechanism previously demonstrated for cellulose nanocrystals (CNCs). ${ }^{23}$ Therefore, other characteristics of CNC-based adhesives such as dependency on substrate type and roughness, humidity, temperature and solvency resistances are similarly anticipated for ChNCs. Thereafter, we investigated adhesive formulations based on the combination of two of the most abundant classes of natural biopolymers, namely, polysaccharides and proteins. Interestingly, testing such biopolymers in the context of lap shear adhesion enables the evaluation of highly brittle constructs and also those that do not readily form materials large enough, e.g., for tensile testing. Therefore, a considerably wider range of material compositions can be explored when compared to other frameworks, e.g., based on films. At a similar areal density, ChNCs presented adhesive performance comparable to that of CNCs. Similarly, HEWL presented high adhesion strength, although it showed substantially lower performance when used in the form of amyloid fibers (both short and long). Strikingly, the interfacial interactions observed between ChNCs and HEWL amyloids resulted in better superstructuring and higher adhesive strengths than those of the individual components alone.

Importantly, from the point of view of wetting and surface energy, optimal superstructure formation for biocolloidal adhesives is developed by systems mostly subjected to capillary forces driving the particle assembly near the edges of the bond. AFM analyses revealed the formation of an intertwined complex between amyloids and chitin, suggesting that the bicomponent systems formed well-ordered, alternating, proteinaceous and chitinous domains. These results suggest that synergetic configurations occur when the more surface-active element does not substantially affect superstructure formation, and mostly contributes to the development of strong interactions with the primary component.

The results of this study point to the high potential of protein-polysaccharide complexes in the formulation of high strength green adhesives. This intimate interaction usually observed in crustaceans, fungi, and arthropods could also be readily exploited, for instance, by using a more gentle extraction process leaving residual proteins on the nanochitins. Moreover, we note that the standardization of recent studies enables the formalization of the interfacial materials chemistry of biopolymers, with the potential to form a library of such interactions for the design of engineered formulations. Lastly, developing our knowledge on interfacial interactions of biopolymeric compounds is anticipated to have important implications in their manufacture and in their assembly, for instance, into functional nanocomposites and into a new generation of adhesives.

\section{Conflicts of interest}

There are no conflicts to declare.

\section{Acknowledgements}

We acknowledge the European Research Council for support under the European Union's Horizon 2020 research and innovation program (ERC Advanced Grant agreement no. 788489, "BioElCell"). Also, we gratefully acknowledge the Academy of Finland's Flagship Programme under project no. 318890 and 318891 (Competence Center for Materials Bioeconomy, FinnCERES). L. G. G. acknowledges funding from the Aalto University School of Chemical Engineering. O. J. R. is grateful for funding support provided by the Canada Excellence Research Chair program. K. J. D. acknowledges funding from the Natural Sciences and Engineering Research Council of Canada (NSERC) Post-doctoral Fellowship program. N. K., S. C. and G. N. also acknowledge funding from the Swiss National Science Foundation (grant no. 200021_192225).

\section{References}

1 M. Cole, P. Lindeque, C. Halsband and T. S. Galloway, Mar. Pollut. Bull., 2011, 62, 2588-2597.

2 R. Geyer, J. R. Jambeck and K. L. Law, Sci. Adv., 2017, 3, e1700782.

3 G. Siqueira, D. Kokkinis, R. Libanori, M. K. Hausmann, A. S. Gladman, A. Neels, P. Tingaut, T. Zimmermann, J. A. Lewis and A. R. Studart, Adv. Funct. Mater., 2017, 27, 1604619.

4 X. Yang, S. K. Biswas, J. Han, S. Tanpichai, M. C. Li, C. Chen, S. Zhu, A. K. Das and H. Yano, Adv. Mater., 2020, 2002264.

5 B. D. Mattos, B. L. Tardy, L. G. Greca, T. Kämäräinen, W. Xiang, O. Cusola, W. L. E. Magalhães and O. J. Rojas, Sci. Adv., 2020, 6, eaaz7328.

6 X. Zhao, J. Zhang and K. Y. Zhu, Extracellular Sugar-Based Biopolymers Matrices, Springer Nature, 2019, pp. 3-56.

7 F. Barthelat, Z. Yin and M. J. Buehler, Nat. Rev. Mater., 2016, 1, 16007.

8 J. Rivera, M. S. Hosseini, D. Restrepo, S. Murata, D. Vasile, D. Y. Parkinson, H. S. Barnard, A. Arakaki, P. Zavattieri and D. Kisailus, Nature, 2020, 586, 543-548. 
9 S. Nikolov, M. Petrov, L. Lymperakis, M. Friák, C. Sachs, H. O. Fabritius, D. Raabe and J. Neugebauer, Adv. Mater., 2010, 22, 519-526.

10 S. O. Andersen, Insect Biochem. Mol. Biol., 2010, 40, 166-178.

11 R. Shelma and C. P. Sharma, Hydrophobically Modified Acylated Chitosan Particles for Drug Delivery Applications: an Overview, CRC Press, 2010.

12 N. Aldred, V. B. S. Chan, K. Emami, K. Okano, A. S. Clare and A. S. Mount, Commun. Biol., 2020, 3, 31.

13 M. Nakano and K. Kamino, Biochemistry, 2015, 54, 826-835.

14 B. Wang and A. Walther, ACS Nano, 2015, 9, 10637-10646.

15 B. L. Tardy, B. D. Mattos, L. G. Greca, T. Kämäräinen, K. W. Klockars and O. J. Rojas, Adv. Funct. Mater., 2019, 29, 1808518.

16 J. G. Fernandez and D. E. Ingber, Adv. Mater., 2012, 24, 480484.

17 G. Nyström, M. P. Fernández-Ronco, S. Bolisetty, M. Mazzotti and R. Mezzenga, Adv. Mater., 2016, 28, 472-478.

18 Q. Luo, C. Hou, Y. Bai, R. Wang and J. Liu, Chem. Rev., 2016, 116, 13571-13632.

19 F. M. Kerton, Y. Liu, K. W. Omari and K. Hawboldt, Green Chem., 2013, 15, 860-871.

20 N. Yan and X. Chen, Nature, 2015, 524, 155-157.

21 C. Lara, J. Adamcik, S. Jordens and R. Mezzenga, Biomacromolecules, 2011, 12, 1868-1875.

22 T. Wu, N. Kummer, K. J. De France, S. Campioni, Z. Zeng, G. Siqueira, J. Dong and G. Nyström, Carbohydr. Polym., 2021, 251, 117021.

23 B. L. Tardy, J. J. Richardson, L. G. Greca, J. Guo, H. Ejima and O. J. Rojas, Adv. Mater., 2020, 32, 1906886.

24 A. Percot, C. Viton and A. Domard, Biomacromolecules, 2003, 4, 12-18.

25 K. J. De France, N. Kummer, Q. Ren, S. Campioni and G. Nyström, Biomacromolecules, 2020, 21, 5139-5147.

26 B. D. Mattos, B. L. Tardy and O. J. Rojas, Biomacromolecules, 2019, 20, 2657-2665.

27 V. P. Santos, N. S. S. Marques, P. C. S. V. Maia, M. A. B. de Lima, L. de O. Franco and G. M. de Campos-Takaki, Int. J. Mol. Sci., 2020, 21, 4290.
28 X. Chen, H. Yang and N. Yan, Chem.-Eur. J., 2016, 22, 1340213421.

29 F. C. Wang and H. A. Wu, Sci. Rep., 2015, 5, 17521.

30 T. Still, P. J. Yunker and A. G. Yodh, Langmuir, 2012, 28, 4984-4988.

31 M. Parsa, S. Harmand, K. Sefiane, M. Bigerelle and R. Deltombe, Langmuir, 2015, 31, 3354-3367.

32 I. Vancea, U. Thiele, E. Pauliac-Vaujour, A. Stannard, C. P. Martin, M. O. Blunt and P. J. Moriarty, Phys. Rev. E: Stat., Nonlinear, Soft Matter Phys., 2008, 78, 041601.

33 A. Gençer, C. Schütz and W. Thielemans, Langmuir, 2017, 33, 228-234.

34 K. W. Klockars, N. E. Yau, B. L. Tardy, J. Majoinen, T. Kämäräinen, K. Miettunen, E. Boutonnet, M. Borghei, J. Beidler and O. J. Rojas, Cellulose, 2019, 26, 491-506.

35 M. Shishehbor, H. Son, M. Nuruddin, J. P. Youngblood, C. Davis and P. D. Zavattieri, J. Mech. Behav. Biomed. Mater., 2021, 118, 104399.

36 J. Zhao, S. Li, R. Zou and A. Yu, Soft Matter, 2012, 8, 10031009.

37 T. Zou, M. H. Sipponen, A. Henn and M. Österberg, ACS Nano, 2021, 15, 4811-4823.

38 S. Beisl, J. Adamcyk, A. Friedl and H. Ejima, Colloid Interface Sci. Commun., 2020, 38, 100306.

39 J. Konnerth, W. Gindl, M. Harm and U. Müller, Holz RohWerkst., 2006, 64, 269-271.

40 J. T. Kim and A. N. Netravali, J. Adhes. Sci. Technol., 2013, 27, 2083-2093.

41 A. D. Roberts, W. Finnigan, P. P. Kelly, M. Faulkner, R. Breitling, E. Takano, N. S. Scrutton, J. J. Blaker and S. Hay, Mater. Today Bio, 2020, 7, 100068.

42 W. Jiang, L. Tomppo, T. Pakarinen, J. A. Sirviö, H. Liimatainen and A. Haapala, BioResources, 2018, 13, 2283-2292.

43 A. Ben Mabrouk, A. Dufresne and S. Boufi, Carbohydr. Polym., 2020, 229, 115504. 